\title{
Upper airway manifestations of granulomatosis with polyangiitis
}

DANIEL S. ALAM, MD; RAHUL SETH, MD; RAJ SINDWANI, MD; ERIKA A. WOODSON, MD; AND KARTHIK RAJASEKARAN, MD

Head and Neck Institute, Cleveland Clinic, Cleveland, $\mathrm{OH}$

\section{ABSTRACT}

Upper airway manifestations, particularly sinonasal manifestations, are encountered frequently in granulomatosis with polyangiitis (GPA). Nasal endoscopy often reveals crusting, friable erythematous mucosa, and granulation. Up to $25 \%$ of patients may have a "saddle-nose" deformity as cartilage destruction worsens. Treatment is often complicated by loss of mucociliary function and necrosis, leading to refractory symptoms. Culture-directed antibiotics, topical antibiotic and saline irrigations, and occasional debridement of adherent crusts can reduce the frequency of sinonasal exacerbations and improve obstructive symptoms. Surgery should be reserved for patients unresponsive to maximal medical therapy. Saddle-nose reconstruction is possible in highly selected patients and can improve nasal breathing and resolve anosmia. Up to $20 \%$ of patients with GPA have subglottic stenosis; patients with respiratory symptoms should undergo laryngoscopy to assess the presence of subglottic narrowing. Although systemic manifestations of GPA are managed by immunosuppressive therapy, most patients with subglottic stenosis of GPA require surgical management (ie, endoscopic dilation, endoscopic or laser excision, surgical resection followed by reconstruction).

T he head and neck are the most common sites of involvement at initial presentation of granulomatosis with polyangiitis (GPA [Wegener's granulomatosis]). Head and neck manifestations occur initially in $73 \%$ of patients, and eventually, up to $92 \%$ of patients with GPA are affected. ${ }^{1}$ Many of these compromise the upper airway. Although treatment is multidisciplinary, the effects on the airway make it important to understand upper airway presentations and treatments. This article examines upper airway disease presentations, their assessment, and their advocated interventions.

\section{DISEASE COURSE}

Because head and neck involvement may be associated with a less aggressive form of GPA, outcomes for patients with predominantly head and neck involvement may be better compared with those who have involvement of other systems. ${ }^{2}$

All authors reported that they have no financial interests or relationships that pose a potential conflict of interest with this article.

doi:10.3949/ccjm.79.s3.04
The natural course of GPA may be indolent or rapidly progressive. Regardless, left untreated, it progresses to a generalized systemic disease that often leads to significant morbidity and likely mortality. Most patients (96\%) achieve remission with immunosuppressive therapy, but nearly half (49\%) have at least one relapse. ${ }^{1}$ For this reason, systemic immunosuppressive medications play a dominant role in systemic and localized head and neck disease control. Patients often require maintenance medications along with additional therapies during disease exacerbation. ${ }^{3}$ Therefore, key partnerships between internists, rheumatologists, and otolaryngologists are paramount in the treatment and follow-up of these patients.

\section{DIAGNOSIS: MAINSTAY IS SEROLOGIC EVALUATION}

The differential diagnosis of GPA includes infection, lymphoproliferative disease (T-cell lymphoma), systemic lupus erythematosus, rheumatoid arthritis, sarcoidosis, and other granulomatous diseases such as eosinophilic GPA (Churg-Strauss syndrome), polyarteritis nodosa, and microscopic polyangiitis. Appropriate diagnosis is critical because treatment of these entities varies drastically.

The mainstay of GPA diagnosis is serologic evaluation for a cytoplasmic pattern of antineutrophil cytoplasmic antibodies (cANCA), which are reactive toward proteinase-3 (PR3) or myeloperoxidase (MPO). Testing for cANCA yields a pooled sensitivity of $91 \%$ and specificity of $99 \%$. Sensitivity falls significantly (63\%) when the disease is in nonacute stages, while the specificity remains high. ${ }^{4}$ These cANCA test characteristics allow a high positive predictive value for this rare disease.

Biopsy is typically reserved for cases in which serologic ANCA testing is nondiagnostic. Biopsy tissue may be readily accessible from the head and neck, but these biopsies may bear significant false-negative rates. ${ }^{4-6}$ Diagnosis requires demonstration of palisading granulomas as vascular or extravascular lesions within the upper respiratory tract tissues. The specific site biopsied from within the head and neck has been shown to influence diagnostic yield, with sinonasal biopsies producing the highest yield.

\section{SINONASAL MANIFESTATIONS}

The nose and paranasal sinuses are the most frequently affected sites in the head and neck, noted in 64\% to $80 \%$ of patients. Additionally, the nose is the only site 
of involvement in $30 \%$ of patients. ${ }^{7}$ Given the high frequency of sinonasal manifestations, GPA should be considered as a potential diagnosis among patients with persistent sinonasal disease.

\section{Pathophysiology and disease course}

The pathophysiologic mechanisms leading to the changes in the sinonasal tract in GPA have not been established. GPA is believed to be an immunologic disease that manifests as a vasculitis of small- and medium-sized vessels. Multiple potential causative factors have been identified, including fibrinoid necrosis of small blood vessels, epithelial granulomas, chronic inflammation, and prior surgical intervention. ${ }^{8,9}$ The acute and chronic inflammation, coupled with the epithelioid granuloma formation, damages adjacent small- to medium-sized vessels. The vasculitis leads to diminished blood flow and subsequent avascular necrosis, which may promote tissue necrosis and bone destruction. This destructive process typically starts in the midseptum supplied by Kiesselbach plexus and in the turbinates. The process then eventually spreads to the paranasal sinuses. ${ }^{8}$

\section{Patient evaluation}

Examination of the nasal cavities is typically performed by rigid or flexible nasal endoscopy and often reveals nasal crusting, friable erythematous mucosa, granulation, and even signs of sinusitis. All or part of the cartilaginous septum may be involved, leading to significant septal defects. As the degree of cartilage destruction increases, nasal dorsal support decreases, leading to a visible depression of the external nose known as a "saddle-nose" deformity, which is present in 23\% of patients with GPA. . $^{710}$

Imaging assessment by computed tomography (CT) is needed to establish disease extent and involvement. Atypical findings may include bony erosion and destruction of the septum and turbinates; erosion of bony partitions within the ethmoid sinuses; neo-osteogenesis of the maxillary, frontal, and sphenoid sinuses; and complete bony obliteration of the maxillary, frontal, and sphenoid sinuses. ${ }^{9,11}$

\section{Clinical presentation}

Sinonasal disease indicates the degree of disease activity. ${ }^{12}$ Clinical findings may vary, but they have a significant impact on quality of life in these patients. ${ }^{13}$ Most patients with active disease present with nasal crusting (69\%), chronic rhinosinusitis (CRS) symptoms (61\%), nasal obstruction (58\%), and serosanguinous nasal discharge (52\%). ${ }^{10}$ Patients may also complain of foul-smelling rhinorrhea, recurrent epistaxis, hyposmia, anosmia, and epiphora (from granulomatous compression or obstruction of the lacrimal system). In a series of 120 patients with GPA, Cannady et al found that four (3.3\%) patients had mucoceles and three $(2.5 \%)$ had orbital pseudotumor. ${ }^{10}$

Any structure in the sinonasal cavity, including mucosa, septum, turbinates, and sinuses proper, may be affected because of the vasculitic involvement of mucosal blood vessels that causes diminished blood flow and subsequent necrosis. The area of the anterior septum supplied by Kiesselbach plexus is the most common site of active nasal disease, which can eventually lead to the common presentation of an anterior nasal septal perforation.

\section{Otologic disease secondary to sinonasal GPA}

Otologic involvement is observed in 19\% to $38 \%$ of patients with GPA. ${ }^{14,15}$ Most patients with GPA who exhibit otologic symptoms have middle ear or mastoid disease. It typically appears as chronic otitis media $(\mathrm{COM})$ with conductive hearing loss. ${ }^{16}$ In most cases, the otologic involvement is secondary to Eustachian tube dysfunction caused by the presence of extensive disease in the nasopharynx.

Additionally, chronic mastoiditis can result from direct mastoid involvement with GPA. Facial nerve palsy secondary to infective bony destruction is a rare but repeatedly reported complication of GPA..$^{14,15}$

Inner ear involvement is a relatively common otologic presentation of GPA. Patients may experience sensorineural hearing loss (SNHL) as well as vertigo, which may mimic Cogan syndrome. Importantly, patients may exhibit inner ear involvement with or without middle ear and mastoid disease. The SNHL observed in patients with GPA may be responsive to steroid or immunosuppressive therapy.

\section{Treatment}

Refractory CRS in GPA is a complex problem for which aggressive surgical intervention is often counterproductive. Unfortunately, traditional medical therapies are also often inadequate to treat progressive sinonasal symptomatology. As the nasal tissue becomes devascularized, loss of normal mucociliary function aggravates the sinus pathology, and clinical symptoms may worsen. Simple antibiotic regimens used to manage uncomplicated sinusitis are often inadequate in these patients. The subsequent progression to frank necrosis in localized regions creates an intranasal foreign body, allowing bacterial colonization, which is often refractory to antibiotics because of the inability of drug tissue penetration into these devascularized nasal structures. ${ }^{12,17}$

Medical management must be tailored to be effective in this complex intranasal milieu. Successful treatment requires a multifaceted and often prolonged treatment course. A high index of suspicion should be maintained for Staphylococcus aureus. As a rule, endoscopically obtained cultures should be used to guide antibiotic selection. Several weeks of culture-directed antibiotics followed by topical antibiotic irrigations (eg, mupirocin irrigations) can be useful to reduce the frequency of sinonasal exacerbations.

Frequent saline irrigations using high-volume, highflow irrigation devices (as opposed to low-volume, lowflow applicators such as nasal spray bottles) can be an 
excellent adjunct to maintenance therapy and are effective in clearing debris and augmenting mucociliary clearance in affected nasal cavities and those with septal perforations. Occasional in-office endoscopic debridement of large crusts adherent to intranasal structures or the edges of a septal perforation can also help to improve obstructive symptoms.

Surgery for refractory cases. Surgery should be reserved for refractory cases unresponsive to maximal medical efforts or those cases with impending complications (ie, mucoceles). Overall, only $16 \%$ of patients with sinonasal GPA required surgical intervention in a large series of 120 patients at our institution. In this series, onethird of all patients had undergone previous functional nasal surgery at an outside institution without resolution of symptoms. Anecdotal evidence suggests that surgery for GPA can contribute to additional scarring and lead to protracted sinonasal symptoms. ${ }^{10,18}$

The decision to perform surgery is individualized and based on severity of the disease process, patient expectations, and surgeon expertise. In our experience at Cleveland Clinic, functional endoscopic sinus surgery in the setting of GPA is a surgical challenge, given extensive alteration of the sinonasal anatomy from previous surgery, prior and ongoing inflammation, chronic crusting, and scarring. Consequently, it has been our practice to employ conservative efforts prior to consideration of surgery. A complete surgical cure is exceedingly rare, and the patient should be counseled about the possible need for revision surgery and ongoing nonsurgical therapies. Meticulous postoperative care with weekly postoperative debridement, saline or antibiotic irrigations, and culture-directed antibiotics, is essential during the early postoperative recovery phase.

Management of epiphora. The most common ophthalmologic findings in patients with GPA include chronic epiphora and orbital pseudotumor. With the advent of advanced endoscopic techniques, the otolaryngologist plays a greater role in the surgical management of these ophthalmologic disease entities. In a series reported by Cannady et al, ${ }^{10}$ endoscopic dacrocystorhinostomy was performed successfully in seven patients, including one revision.

Nasal reconstruction for saddle-nose deformity: effective in selected patients. The progressive loss of septal support that occurs with the enlarging anterior septal perforation often results in significant collapse of the cartilaginous midvault of the nose. The tip cartilages in turn also begin to lose projection, resulting in a shortened nose with the characteristic saddle-nose deformity. The psychologic impact of this disfiguring facial abnormality is significant. The loss of midvault support also results in worsening nasal obstruction and increases the incidence of anosmia as the superior nasal vault becomes obstructed. For these reasons, patients often seek referral for potential reconstruction.
Despite the potential benefits, the general consensus in the medical community has been that surgical procedures on the nose should be avoided in GPA patients. ${ }^{17}$ Most nasal destruction in these patients is the consequence of poor tissue perfusion from the active vasculitis. Poor wound healing, reconstructive graft resorption, and worsening necrosis have been observed in patients who have undergone ill-advised surgical procedures.

These poor outcomes do not, however, preclude the potential for safe and effective surgical intervention. In three small published series, good surgical outcomes were achieved but the procedures were done in very highly selected patients and were modified to address the specific clinical issues seen in GPA patients. ${ }^{19-21}$ The critical step in achieving a good outcome is working closely with the patient's rheumatologist to identify an appropriate clinical window during which the patient's disease process is in a period of relative remission. The second major factor is to modify the surgical techniques to take into account the very poor vascular framework of the recipient nasal bed.

Outcomes include structural correction of the saddle nose, improved nasal breathing, resolution of anosmia, and improved nasal hygiene, leading to improved quality of life (Figure).

Management of COM. Because the COM in patients with GPA is frequently secondary to nasopharyngeal disease, systemic control of GPA is the first priority. Systemic control is also the first-line treatment for patients with mixed or sensorineural hearing loss, or with vertigo. For continued or symptomatic middle ear effusions that do not resolve with systemic therapy, placement of a ventilation tube may be considered. In patients with significant hearing loss, hearing amplification devices may be warranted. ${ }^{15,22}$ Cochlear implant devices in GPA patients are experimental and may pose undue risks of meningitis.

\section{SUBGLOTTIC STENOSIS AND TRACHEAL MANIFESTATIONS}

Subglottic stenosis affects $10 \%$ to $20 \%$ of patients with GPA. ${ }^{1,23,24}$ Because of its potential life-threatening airway complications, patients should be carefully assessed for this disease manifestation. It may be the only manifestation of GPA or may be part of a spectrum of other disease manifestations. Therefore, the work-up for subglottic stenosis of unknown etiology should always include an evaluation for GPA.

\section{Pathophysiology and disease course}

The etiology of subglottic stenosis in GPA is not well understood. Theories primarily involve the vulnerability of the subglottic tissues to damage, chronic inflammation, and scarring. ${ }^{25}$ The combination of vasculitis in the setting of active inflammation may synergistically 
produce a hyperactive reparative mechanism in GPA patients that leads to cartilaginous fibrotic scarring and stenosis. Wound healing can be divided into the phases of inflammation, proliferation, and remodeling. An imbalance or exaggerated response of any of these levels (and likely all) produces an abnormal healing response. ${ }^{26}$ Similarly, each of these phases may be targeted to improve the healing process.

\section{Patient evaluation}

The presence of subglottic stenosis must be considered in a GPA patient with respiratory symptoms. As part of the routine initial evaluation, an office-based nasopharyngeal/laryngeal endoscopy using a flexible laryngoscope should be performed to assess the presence and severity of luminal airway narrowing. Flexible laryngoscopy reveals a circumferential narrowing of the subglottis. The stenotic tissue may vary from friable with erythematous and inflamed mucosa to a rigid mature fibrotic band, depending on the inflammatory state of the stenosis. ${ }^{18,27}$

Subclinical stenosis may be identified with routine endoscopy. An appropriate baseline is needed to follow the progression of disease and to adjust the timing of any potential intervention. The ability to digitally record a patient's examination allows further tracking of disease and is commonly used in our practices.

Although flexible fiberoptic examination is critical in diagnosis and follow-up, intraoperative direct laryngoscopy using rigid laryngoscopes and telescopes provides the optimum view of the subglottis. In particular, this view provides greater information on the length and degree of the stenosis and allows evaluation of potential stenotic segments in the inferior trachea.

Spiral CT with 3-dimensional reconstruction of the laryngotracheal lumen and virtual bronchoscopy may provide information that complements laryngoscopy. CT may permit assessment of the entire tracheobronchial pathway. Because $15 \%$ to $55 \%$ of GPA patients have additional bronchial stenotic segments, assessment of the entire airway is important. ${ }^{28,29}$

\section{Clinical presentation}

Diagnosis of GPA in patients younger than 20 years is associated with the development of subglottic stenosis. ${ }^{23,30}$ The GPA patient with subglottic stenosis may or may not have other active systemic symptoms. The efficacy of systemic therapy often does not correlate with the degree of subglottic stenosis. Importantly, when systemic disease enters remission, the subglottic stenosis may remain due to residual scarring of the subglottis. ${ }^{31}$

Patients with subglottic stenosis may present with hoarseness, cough, wheeze, stridor, or dyspnea on exertion. ${ }^{27,32}$ The stridor and wheeze may be confused with the wheeze of asthma, often leading to misdiagnosis. ${ }^{17}$

Subglottic stenosis likely begins at a small degree and increases gradually, allowing the patient to adjust his
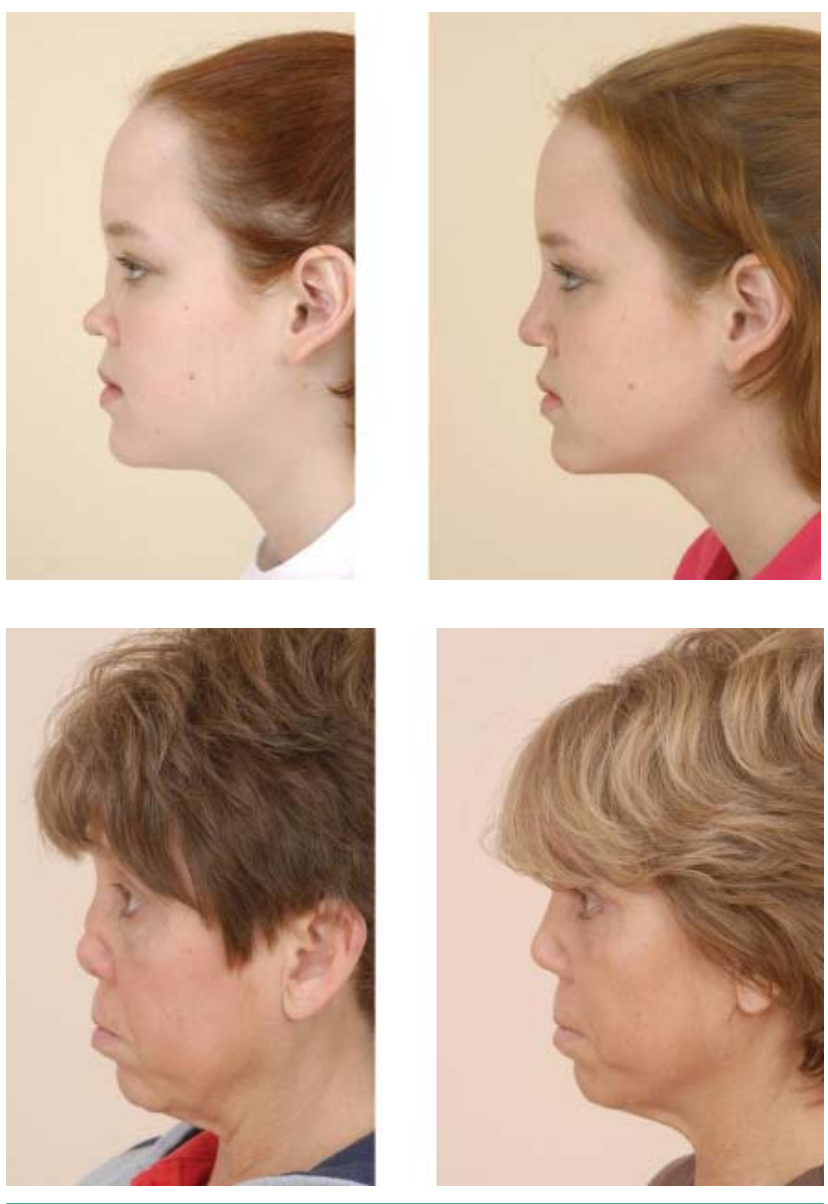

FIGURE. Well-timed surgical intervention can correct the saddlenose deformity, improve nasal breathing and hygiene, and resolve anosmia.

or her breathing pattern until a critical stenotic airway area is reached. Typically, and dependent on their pulmonary health, patients are asymptomatic until about $75 \%$ airway stenosis (60\% in children). ${ }^{33,34}$ At this point, symptoms may become evident and correlate with the degree of stenosis, ranging from cough and mild shortness of breath to life-threatening stridor and obstruction. Importantly, as the airway caliber narrows, mucous plugging becomes a greater concern, as it can cause acute stridulous exacerbations and airway obstruction.

A significant proportion of patients with GPA who have subclinical asymptomatic stenosis may not receive laryngeal examination. Patients who have suspicious clinical histories should be referred for evaluation of subglottic stenosis prior to symptom worsening.

Patients with significant (approximately 80\%) stenosis can present with respiratory symptoms that may be life-threatening. Because airway management in this setting is substantially more difficult, the goal should be to obtain a diagnosis and perform intervention before this advanced presentation develops.

Pauzner et al described a possible association between 
GPA tracheal stenosis and pregnancy. ${ }^{35}$ Women of childbearing age who have GPA should be counseled about this possible association and the need for close follow-up during the partum and postpartum periods.

\section{Treatment is controversial}

The treatment of subglottic stenosis of GPA requires multidisciplinary management by the rheumatologist, otolaryngologist, and pulmonologist. Systemic manifestations of disease are managed by immunosuppressive therapy, but up to $80 \%$ of patients may require surgical management of subglottic stenosis, and the remaining $20 \%$ will respond to systemic medical therapy. ${ }^{22,23,36,37}$ Overall, the treatment of this disease is controversial and varies by center. The therapeutic arsenal consists of conventional immunosuppressive therapy, endoscopic dilation, endoscopic or laser excision, and surgical resection of the stenotic segment followed by reconstruction.

Tracheotomy. Historically, tracheotomies were performed in approximately one-half of patients with airway manifestations of GPA when the patient had active disease or when airway patency could not be adequately maintained. Most of these patients were eventually decannulated..$^{23,25}$ At present, tracheotomy is performed infrequently and is reserved for patients who have either a severely tenuous airway (with tracheotomy the only safe option available to obtain a secure airway) or who express a preference for tracheotomy. In a recent study by Hoffman et $\mathrm{al}^{38}$ tracheotomy was avoided in 21 patients through the use of stenosis dilation procedures.

Dilation. Endoscopic subglottic dilation is the currently advocated method of treatment, and has shown promising results. In two studies with a total of 41 GPA patients who were able to avoid tracheotomy and open surgical procedures, $24 \%$ underwent decannulation of previously placed tracheotomies and $24 \%$ required only one procedure at an average follow-up of 3.4 and 5 years per study. In these studies, the technique of intralesional corticosteroid with mechanical dilation (ILCD) was performed. ${ }^{31,36,38}$

\section{Preferred: Dilation plus medical therapy}

Because of the inflammatory etiology of this condition, surgical intervention has the risk of potentially worsening the stenosis. However, combining dilation of the stenosis with aggressive local medical treatment to prevent scar formation and cellular proliferation has been shown to be effective and safe. This treatment modality was recently recommended as the preferred therapy based on a number of relatively small clinical trials for subglottic stenosis, without the benefit of large controlled trials.

Our patient population consists of two subsets: (1) those who respond well to ILCD and systemic medical therapy, requiring a minimal number of dilations before no longer needing procedures because of a possible "burn out" of the subglottic disease, and (2) those who continue to have recurrence of stenosis, requiring repeat ILCD. The latter group requires close long-term observation.

To counter the effects of the exaggerated healing reaction of inflammation (early) and proliferation (late) following injury, two medications are applied to the area of repaired stenosis. The stenotic lesion is first injected submucosally with a long-acting corticosteroid suspension such as methylprednisolone. The solution is injected along the submucosal-perichondrial plane. Incisions are made in a star-like fashion, employing sharp metal microlaryngeal blades or, less commonly, the carbon dioxide laser. These incisions release the constricting stenotic ring and break it up, widening the diameter of the airway and simultaneously preserving islands of intact mucous membrane between the incisions. This epithelium is intended to regenerate and resurface the expanded lumen. Progressive serial dilations are performed using semirigid, flexible, smooth dilators or high-pressure balloon dilation. The next stage involves repeated topical applications of mitomycin- $\mathrm{C}$ to further inhibit fibrosis and restenosis by inhibiting cellular proliferation of the vigorous injury cycles of these lesions. Application of mitomycin-C to the dilated area of a laryngotracheal stenosis has been associated with a decreased rate of stenosis relapse. ${ }^{39}$

Our group at Cleveland Clinic has never used laser surgery alone without dilation on the subglottic stenosis caused by GPA. Incidentally, patients treated with laser surgery in other institutions prior to their referral to the Cleveland Clinic have developed complicating secondary stenoses that required more extensive surgical intervention to overcome the severe secondary superimposed damage. In theory, use of the laser may create unnecessary thermal injury that likely worsens local damage. These patients required laryngotracheal reconstructive procedures or had to undergo establishment of permanent tracheotomies.

\section{CONCLUSION}

Granulomatosis with polyangiitis is a rare disease that may manifest in multiple areas of the head and neck. Careful attention to diagnosis and management is criti$\mathrm{cal}$, as these patients tend to have progressive disease with debilitating sequelae. The rheumatologist, otolaryngologist, and internist should identify patients with any constellation of symptoms that may be typical of GPA. A collaborative effort to diagnose, treat, and follow these patients is paramount to successful disease management.

\section{REFERENCES}

1. Hoffman GS, Kerr GS, Leavitt RY, et al. Wegener granulomatosis: an analysis of 158 patients. Ann Intern Med 1992; 116:488-498.

2. Mahr A, Girard T, Agher R, Guillevin L. Analysis of factors predictive of survival based on 49 patients with systemic Wegener's granulomatosis and prospective follow-up. Rheumatology (Oxford) 2001; 40:492-498.

3. Wung PK, Stone JH. Therapeutics of Wegener's granulomatosis. 
Nat Clin Pract Rheumatol 2006; 2:192-200.

4. Rao JK, Weinberger M, Oddone EZ, Allen NB, Landsman P, Feussner JR. The role of antineutrophil cytoplasmic antibody (c-ANCA) testing in the diagnosis of Wegener granulomatosis: a literature review and meta-analysis. Ann Intern Med 1995; 123:925-932.

5. Devaney KO, Travis WD, Hoffman G, Leavitt R, Lebovics R, Fauci AS. Interpretation of head and neck biopsies in Wegener's granulomatosis: a pathologic study of 126 biopsies in 70 patients. Am J Surg Pathol 1990; 14:555-564.

6. Jennings CR, Jones NS, Dugar J, Powell RJ, Lowe J. Wegener's granulomatosis-a review of diagnosis and treatment in 53 subjects. Rhinology 1998; 36:188-191.

7. McDonald TJ, DeRemee RA. Wegener's granulomatosis. Laryngoscope 1983; 93:220-231.

8. Lloyd G, Lund VJ, Beale T, Howard D. Rhinologic changes in Wegener's granulomatosis. J Laryngol Otol 2002; 116:565-569.

9. Yang C, Talbot JM, Hwang PH. Bony abnormalities of the paranasal sinuses in patients with Wegener's granulomatosis. Am J Rhinol 2001; 15:121-125

10. Cannady SB, Batra PS, Koening C, et al. Sinonasal Wegener granulomatosis: a single-institution experience with 120 cases. Laryngoscope 2009; 119:757-761.

11. Grindler D, Cannady S, Batra PS. Computed tomography findings in sinonasal Wegener's granulomatosis. Am J Rhinol Allergy 2009; 23:497-501.

12. Hughes RG, Drake-Lee A. Nasal manifestations of granulomatous disease. Hosp Med 2001; 62:417-421.

13. Srouji IA, Andrews P, Edwards C, Lund VJ. General and rhinosinusitis-related quality of life in patients with Wegener's granulomatosis. Laryngoscope 2006; 116:1621-1625.

14. Kornblut AD, Wolff SM, deFries HO, Fauci AS. Wegener's granulomatosis. Laryngoscope 1980; 90:1453-1465.

15. McCaffrey TV, McDonald TJ, Facer GW, DeRemee RA. Otologic manifestations of Wegener's granulomatosis. Otolaryngol Head Neck Surg 1980; 88:586-593.

16. Bradley PJ. Wegener's granulomatosis of the ear. J Laryngol Otol 1983; 97:623-626

17. Rasmussen N. Management of the ear, nose, and throat manifestations of Wegener granulomatosis: an otorhinolaryngologist's perspective. Curr Opin Rheumatol 2001; 13:3-11.

18. Erickson VR, Hwang PH. Wegener's granulomatosis: current trends in diagnosis and management. Curr Opin Otolaryngol Head Neck Surg 2007; 15:170-176.

19. Congdon D, Sherris DA, Specks U, McDonald T. Long-term followup of repair of external nasal deformities in patients with Wegener's granulomatosis. Laryngoscope 2002; 112:731-737.

20. Duffy FJ Jr, Rossi RM, Pribaz JJ. Reconstruction of Wegener's nasal deformity using bilateral facial artery musculomucosal flaps. Plast Reconstr Surg 1998; 101:1330-1333.

21. Shipchandler TZ, Chung BJ, Alam DS. Saddle nose deformity reconstruction with a split calvarial bone $\mathrm{L}$-shaped strut. Arch Facial Plast Surg 2008; 10:305-311.

22. Hernández-Rodríguez J, Hoffman GS, Koening CL. Surgical interventions and local therapy for Wegener's granulomatosis. Curr Opin Rheumatol 2010; 22:29-36.

23. Lebovics RS, Hoffman GS, Leavitt RY, et al. The management of subglottic stenosis in patients with Wegener's granulomatosis.
Laryngoscope 1992; 102:1341-1345.

24. Waxman J, Bose WJ. Laryngeal manifestations of Wegener's granulomatosis: case reports and review of the literature. J Rheumatol $1986 ; 13: 408-411$

25. Maronian NC, Azadeh H, Waugh P, Hillel A. Association of laryngopharyngeal reflux disease and subglottic stenosis. Ann Otol Rhinol Laryngol 2001; 110:606-612.

26. Diegelmann RF, Evans MC. Wound healing: an overview of acute, fibrotic and delayed healing. Front Biosci 2004; 9:283-289.

27. Gubbels SP, Barkhuizen A, Hwang PH. Head and neck manifestations of Wegener's granulomatosis. Otolaryngol Clin North Am 2003;36:685-705

28. Polychronopoulos VS, Prakash UB, Golbin JM, Edell ES, Specks U. Airway involvement in Wegener's granulomatosis. Rheum Dis Clin North Am 2007; 33:755-775.

29. Daum TE, Specks U, Colby TV, et al. Tracheobronchial involvement in Wegener's granulomatosis. Am J Respir Crit Care Med 1995; 151:522-526.

30. Rottem M, Fauci AS, Hallahan CW, et al. Wegener granulomatosis in children and adolescents: clinical presentation and outcome. J Pediatr 1993; 122:26-31

31. Eliachar I, Chan J, Akst L. New approaches to the management of subglottic stenosis in Wegener's granulomatosis. Cleve Clin J Med 2002; 69(suppl 2):SII149-SII151.

32. Solans-Laqué R, Bosch-Gil J, Canela M, Lorente J, Pallisa E, Vilardell-Tarrés M. Clinical features and therapeutic management of subglottic stenosis in patients with Wegener's granulomatosis. Lupus 2008; 17:832-836.

33. Sandu K, Monnier P. Cricotracheal resection. Otolaryngol Clin North Am 2008; 41:981-998.

34. Brouns M, Jayaraju ST, Lacor C, De Mey J, et al. Tracheal stenosis: a flow dynamics study [published online ahead of print November 30, 2006]. J Appl Physiol 2007; 102:1178-1184. doi: 10.1152/ japplphysiol.01063.2006

35. Pauzner R, Mayan H, Hershko E, Alcalay M, Farfel Z. Exacerbation of Wegener's granulomatosis during pregnancy: report of a case with tracheal stenosis and literature review. J Rheumatol 1994; 21:1153-1156

36. Langford CA, Sneller MC, Hallahan CW, et al. Clinical features and therapeutic management of subglottic stenosis in patients with Wegener's granulomatosis. Arthritis Rheum 1996; 39:1754-1760.

37. Schokkenbroek AA, Franssen CF, Dikkers FG. Dilatation tracheoscopy for laryngeal and tracheal stenosis in patients with Wegener's granulomatosis [published online ahead of print November 14, 2007]. Eur Arch Otorhinolaryngol 2008; 265:549-555. doi: 10.1007/s00405-007-0518-3

38. Hoffman GS, Thomas-Golbanov CK, Chan J, Akst LM, Eliachar I. Treatment of subglottic stenosis, due to Wegener's granulomatosis, with intralesional corticosteroids and dilation. J Rheumatol 2003; 30:1017-1021.

39. Smith ME, Elstad M. Mitomycin C and the endoscopic treatment of laryngotracheal stenosis: are two applications better than one? Laryngoscope 2009; 119:272-283.

Correspondence: Daniel S. Alam, MD, Head and Neck Institute, Cleveland Clinic, 9500 Euclid Avenue, A71, Cleveland, OH 44195; alamd@ccf.org 


\title{
Renal disease in small-vessel vasculitis
}

\author{
KIRSTEN de GROOT, MD \\ Head, Department of Nephrology/Rheumatology, Klinikum Offenbach GmbH, KfH Nierenzentrum Offenbach, Offenbach, Germany
}

\section{ABSTRACT}

Glomerulonephritis (GN) is a common manifestation of the antineutrophil cytoplasmic antibody-associated systemic vasculitides (AASV), which include granulomatosis with polyangiitis and microscopic polyangiitis. The level of renal involvement at presentation is highly predictive of survival and should be assessed early so that kidney function can be preserved. AASV patients with urinary sediment but normal function have a twofold greater risk of death than those with no renal involvement. Those with impaired renal function at diagnosis have a fivefold greater risk of death. Renal vasculitis is most prevalent in older patients, who have more severe disease and poorer prognoses. Renal biopsy not only establishes diagnosis but provides information on severity of renal-function impairment and prognosis. Induction of remission with cyclophosphamide is standard treatment. For patients with crescentic, rapidly progressive $\mathrm{GN}$, adjunctive plasma exchange can promote renal recovery. Renal failure occurs in one-fourth of AASV patients after 3 to 4 years; $60 \%$ of patients receiving dialysis for acute $\mathrm{GN}$ can recover independent renal function. Renal transplant patients with vasculitis fare as well as renal transplant patients without vasculitis. Lastly, renal vasculitis is an independent risk factor for cardiovascular events.

N ecrotizing glomerulonephritis (GN) is the classical renal manifestation of the smallvessel vasculitides, which include granulomatosis with polyangiitis (GPA [Wegener's granulomatosis]), microscopic polyangiitis (MPA), and eosinophilic GPA (Churg-Strauss syndrome). MPA is pauci-immune (lacks antibody depositions) and causes focal segmental necrotizing GN. Other small-vessel vasculitides are Henoch-Schönlein purpura, which features nephritis from immunoglobulin A-dominant immune deposits, and essential cryoglobulinemic vasculitis, in which cryoglobulin immune deposits lead to membranoproliferative GN. ${ }^{1}$

Renal involvement occurs in $25 \%$ to $75 \%$ of patients with antineutrophil cytoplasmic antibody (ANCA)associated systemic vasculitis (AASV), with the higher percentage reflecting patients who first present to a nephrologist rather than a rheumatologist. Roughly 35\% are dialysis-dependent, mainly those who are elderly or proteinase-3- or myeloperoxidase-ANCA-positive. Induction of remission through immunosuppression allows $50 \%$ to $60 \%$ of dialysis-dependent patients to recover independent renal function. ${ }^{2,3}$

\section{PROGNOSTIC SIGNIFICANCE OF RENAL INVOLVEMENT}

Patients should be assessed at the earliest opportunity for renal involvement as it is highly predictive of survival. Diagnosis allows immunosuppressive treatment to be started early, when kidney function may still be preserved.

Reinhold-Keller et $\mathrm{al}^{3}$ examined survival by level of renal involvement at diagnosis in 155 patients with GPA who were followed for a median of 7 years. Survival in those with normal renal function, but with nephritic urinary sediment at diagnosis, declined over time compared with patients who had no renal involvement at diagnosis, with a more than twofold greater risk of death (hazard ratio [HR], 2.41; 95\% confidence interval [CI] 0.53-11.06). Patients who had impaired renal function at diagnosis had a fivefold greater risk of death (HR, 5.42; 95\% CI 1.76-16.68).

Maximum serum creatinine in the first month of treatment is also highly predictive of survival. An outcome analysis followed 80 patients with AASV and renal involvement for a median of 46.7 months. ${ }^{4}$ Patients were divided equally into groups by maximum serum creatinine levels after the first month of treatment: less than $299 \mu \mathrm{mol} / \mathrm{L}, 299$ to $582 \mu \mathrm{mol} / \mathrm{L}$, and greater than $582 \mu \mathrm{mol} / \mathrm{L}$. All patients were treated for induction of remission with cyclophosphamide and oral corticosteroids. Survival was significantly worse in patients who had the highest maximum serum creatinine in the first month $(P=.025)$.

Pooled prospective data from four European Vasculitis Study Group (EUVAS) trials of 535 patients with AASV and follow-up of 5.2 years found a mortality ratio of 2.6 (95\% CI, 2.2-3.1) compared with matched subjects from the general population. ${ }^{5}$ Stage 5 chronic kidney disease (glomerular filtration rate $<15 \mathrm{~mL} / \mathrm{min}$ ) was a significant negative prognostic determinant of survival in these trials.
Dr. de Groot reported that she has no financial interests or relationships that pose a potential conflict of interest with this article.

This article was developed from an audio transcript of Dr. de Groot's presentation at the "New Directions in Small-Vessel Vasculitis: ANCA, Target Organs, Treatment, and Beyond" symposium held at Cleveland Clinic on May 4, 2011.
The transcript was formatted and edited by Cleveland Clinic Journal of Medicine staff for clarity and conciseness, and was then reviewed, revised, and approved by Dr. de Groot.

doi:10.3949/ccjm.79.s3.05 


\section{A DISEASE OF AGING}

Renal vasculitis is most prevalent in people aged 50 years and older and often occurs in those aged 70 years and older. $^{6}$ Because the very old may not have extrarenal symptoms, it is necessary to maintain a high index of suspicion for AASV and measure urinary sediment and renal function in this age group.

Older patients also present with more severe renal disease and have a poorer prognosis. Harper and Savage compared presentation and outcomes of patients aged 65 years and older with renal AASV with those of patients younger than 65 years. ${ }^{7}$ Older patients had more severe renal failure than did younger patients (serum creatinine 657 vs $470 \mu \mathrm{mol} / \mathrm{L}$, respectively; $P<.001$ ), and this did not appear to be associated with delayed diagnosis. Survival was worse in those with serum creatinine levels greater than $400 \mu \mathrm{mol} / \mathrm{L}$ irrespective of age; however, when comparing younger and older groups with similar renal insufficiency, older patients were more likely to progress to end-stage renal failure $(P=.039)$, survival was worse $(P=.016)$, and death occurred earlier. ${ }^{7}$

\section{- HISTOPATHOLOGIC CLASSIFICATION}

An international working group of renal pathologists ${ }^{8}$ developed pathologic classifications for rapidly progressive GN caused by AASV: focal $(\geq 50 \%$ normal glomeruli), crescentic ( $\geq 50 \%$ glomeruli with cellular crescents), mixed (no predominant glomerular feature), and sclerotic ( $\geq 50 \%$ globally sclerotic glomeruli). These correspond to the order of severity of renal-function impairment. A study of 100 biopsies from patients with ANCA-associated GN found that the classifications at presentation closely correlated with outcomes. ${ }^{8}$

Patients with sclerotic GN had the worst renal function initially, with little improvement at 5 years; hence, immunosuppression is of little value if the kidney is more than $50 \%$ sclerotic. Patients with focal disease who had good renal function initially were found to retain good function after 5 years of treatment. Patients in the crescentic class present with rapidly progressive GN and very poor renal function. However, they were found to improve considerably after 5 years and had good recovery $(P=.001)$. Presenting disease manifestations within the kidney are of diagnostic as well as prognostic value.

\section{TREATMENT OF AASV}

\section{Lower dosage of cyclophosphamide}

Standard immunosuppressive treatment for vasculitis is oral cyclophosphamide, $2 \mathrm{mg} / \mathrm{kg}$ per day. To reduce toxic effects and amount of the drug used, we tested whether a pulse dose could induce remission. In the EUVAS randomized trial of oral versus pulse cyclophosphamide (the CYCLOPS study), ${ }^{9} 149$ AASV patients with renal involvement received either pulse cyclophosphamide,
$15 \mathrm{mg} / \mathrm{kg}$ every 2 to 3 weeks, or daily oral cyclophosphamide, $2 \mathrm{mg} / \mathrm{kg}$ per day, plus prednisone. The groups did not differ in time to remission (HR, 1.098) or in proportion of patients who had achieved remission at 9 months $(88.1 \%$ vs $87.7 \%)$. The pulse-dose group needed half the amount of drug to achieve remission compared with the oral-dose group. Pulse dosing is currently the preferred method in Europe, where doses are administered in the clinic rather than at home.

In a 4.3-year follow-up, twice as many patients relapsed in the pulse-dose group compared with the oral group (HR, 0.50; $P=.029$ ), but there was no difference between groups in renal function $(P=.82)$, end-stage renal disease (ESRD), or death. ${ }^{10}$ It should be borne in mind that there is a tendency to overtreat. An analysis of four EUVAS trials found a risk of mortality of $11 \%$ in the first year; $59 \%$ of these were due to treatmentrelated adverse events. ${ }^{11}$

Despite being prone to renal failure and infectious complications, elderly patients with ANCA-associated GN who do not have ESRD fare better with immunosuppressive therapy than without in terms of progression and survival. Cyclophosphamide dosage should be reduced in these patients.

\section{Plasma exchange}

In crescentic disease and rapidly progressing renal failure, plasma exchange (PE) with albumin reduces circulating antibodies by up to $60 \%$ and promotes renal recovery. The MEPEX trial compared the addition of either PE or intravenous methylprednisolone (MEP) to oral cyclophosphamide and prednisolone in 137 newly diagnosed AASV patients with severe crescentic GN (serum creatinine $>500 \mu \mathrm{mol} / \mathrm{L}$ ). ${ }^{12}$ Two-thirds of the group were oliguric. After 3 months, $69 \%$ of PE-treated compared with $49 \%$ of MEP-treated patients were alive and had achieved independent renal function $(P=.02)$. There was also a reduction in risk for ESRD of $24 \%$ at 12 months in the PE versus MEP group. Mortality was similarly high in both groups, however; roughly one-third had died by 12 months, reflecting the higher rates of complications in this older-aged group (median, 66 years).

For patients undergoing PE who have a sudden drop in hemoglobin, gastrointestinal bleeding is only one possible underlying condition. The patient may have bleeding into the pulmonary parenchyma, and computed tomography of the lung should be performed. This is more likely to occur when plasma is exchanged with albumin rather than fresh frozen plasma.

\section{Renal replacement therapy}

End-stage renal disease occurs in approximately 25\% of patients 3 to 4 years after they present with AASV. Renal-limited disease occurs most often in those with MPA. When there is active rather than sclerotic disease but irreversible renal failure is suspected, immunosuppression can be tried for 3 months. If there is no 


\section{CLINICAL CASE: Confusing presentation and differential diagnosis}

\begin{abstract}
A 73-year-old man had a flulike illness with headache, myalgia, and fever that did not respond to treatment with penicillin. He took up to 2 grams of nonsteroidal antiinflammatory drugs (NSAIDs) daily. He was hospitalized with epistaxis and melena and transferred to our care. The patient became oliguric, passing only a few hundred $\mathrm{mL}$ urine per day, with serum creatinine greater than $700 \mu \mathrm{mol} / \mathrm{L}$. His history was unremarkable except for chronic obstructive pulmonary disease and tobacco use. The preliminary diagnosis was acute oliguric kidney failure.
\end{abstract}

\begin{tabular}{ll} 
Laboratory test results on admission \\
\hline Blood values & Urine values \\
Serum creatinine, $767 \mu \mathrm{mol} / \mathrm{L}$ & Dipstick: \\
Urea, $31 \mathrm{mmol} / \mathrm{L}$ & Protein, +++ \\
Electrolytes, normal & Erythrocytes, ++++ \\
Hemoglobin, $8.7 \mathrm{~g} / \mathrm{dL}$ & Leukocytes, + \\
7.8 leukocytes $/ \mathrm{nL}$ & Nitrite, negative \\
89 thrombocytes $/ \mathrm{nL}$ & Sediment: \\
C-reactive protein, $129 \mathrm{mg} / \mathrm{L}$ & Erythrocytes, $>200$ \\
Albumin, $23 \mathrm{mg} / \mathrm{dL}$ & No acanthocytes \\
Total protein, $58 \mathrm{mg} / \mathrm{dL}$ & Leukocytes, 3-5 \\
Lactate dehydrogenase, $290 \mathrm{U} / \mathrm{L}$ & No cylinders, epithelial cells, \\
Scattered fragmentocytes & bacteria, fungi, or crystals \\
\end{tabular}

The patient had very high serum creatinine and low hemoglobin levels. He also had low thrombocytes-an unusual finding in vasculitis. In addition, he had low albumin and elevated lactate dehydrogenase levels. Differential diagnosis needed to account for acute renal failure, antibioticresistant fever, and possible otolaryngologic disease. Results of antineutrophil cytoplasmic antibody (ANCA) testing would not arrive for several days.

response, improvement in renal function is unlikely and immunosuppressive treatment is continued only for extrarenal disease. Patients with ESRD can be treated with hemodialysis, peritoneal dialysis at home, or kidney transplant.

Lionaki et $\mathrm{al}^{13}$ described the rate of relapse in AASV patients before and after kidney dialysis compared with that in AASV patients with preserved renal function. Over a median of 40 months, 136 of 523 patients progressed to ESRD. Rate of relapse of vasculitis was significantly lower for the patients on chronic dialysis (0.08 episodes per person-year) than for the same patients before dialysis ( 0.2 episodes) and for the patients with preserved

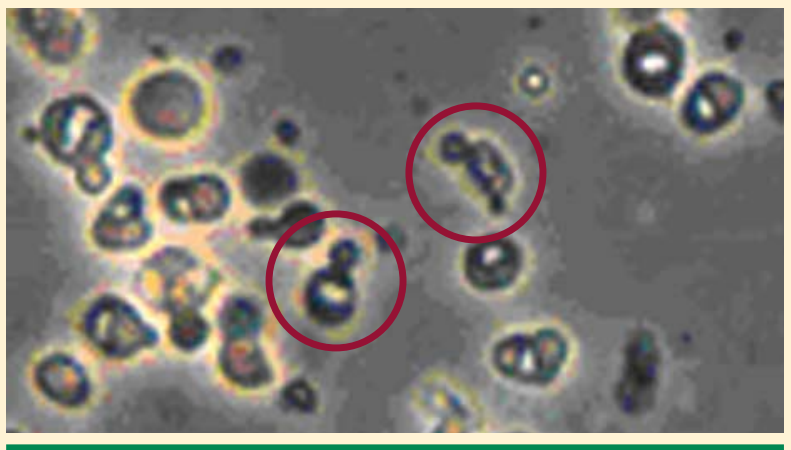

FIGURE 1. Urinary sediment under a polarization microscope. Urinary erythrocytes with the shape of mouse ears (circled) are a sign of erythrocyturia due to glomerular damage.

A urine dipstick test showed proteinuria, corresponding to very low albumin and erythrocyturia. Sediment analysis did not reveal the typical acanthocytes and cylinders. Instead, erythrocytes resembling mouse ears were seen (Figure 1).

Mouse ears occur when erythrocytes pass through the glomerular basement; they indicate an inflammatory disorder of the glomerular barrier and are highly suggestive of glomerulonephritis (GN), which is confirmed by renal biopsy. A more typical presentation includes active urinary sediment with a variable proteinuria of less than 3 grams per day. Arterial hypertension, oliguria, and reduced glomerular filtration rate may be present.

\section{Differential diagnosis}

Differential diagnoses included ANCA-associated systemic vasculitis (AASV), cryoglobulinemic vasculitis, hemolytic uremic syndrome, interstitial nephritis from NSAID use, relapsing polychondritis with GN in systemic lupus erythematosis, and anti-glomerular basement membrane (GBM) antibody disease.

Ultrasound showed bilaterally enlarged kidneys, echogenic parenchyma, hypoechoic medullar pyramids, no

renal function ( 0.15 episodes). Infections, an important cause of death, were twice as frequent for patients on dialysis and maintenance immunosuppression.

Weidanz et $\mathrm{al}^{14}$ reported on a retrospective case series that examined whether immunosuppressive therapy with its risk of infection is beneficial for vasculitis patients on dialysis. They retrospectively examined 46 cases of AASV over 30 years and found that the patients with ESRD received less immunosuppression, but their rate of infection was twice that of pre-ESRD patients, and mortality quadrupled while on dialysis. The mode of dialysis did not affect survival, however. These results may support early discontinuation of immunosuppressive 


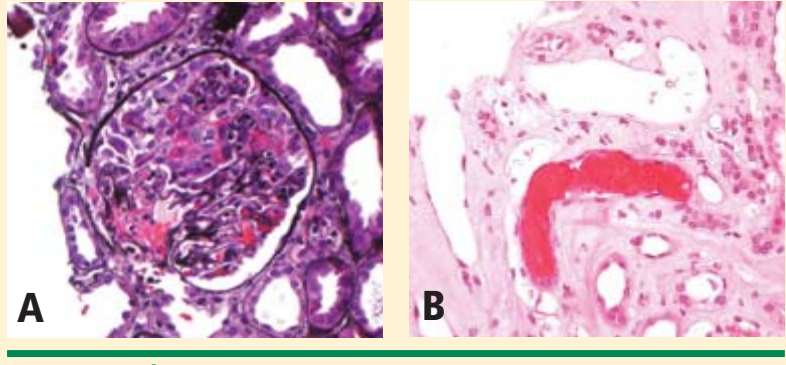

FIGURE 2. (A) Areas of necrosis did not encompass the glomerulus. (B) Tubules packed with erythrocytes.

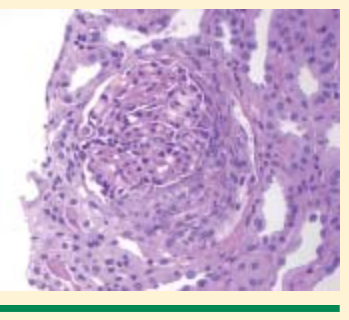

FIGURE 3. Formation of a cellular crescent. stone or tumor, and hyperhydration. The patient had atrial fibrillation on electrocardiography due to the inflammatory process. Echocardiography revealed dilated cardiac cavities, regular ejection fraction, and no hypertrophy; in addition, moderate atrial

insufficiency, mitral insufficiency, and pulmonary arterial hypertension were observed.

\section{Interpreting histopathology in AASV}

Renal biopsy is required to establish the diagnosis of rapidly progressive GN caused by AASV.

Focal segmental GN. Jones's silver stain of GBM showed areas of necrosis that did not encompass the whole glomerulus (ie, segmental) (Figure 2A).

Tubules were packed with erythrocytes (Figure 2B). When expelled in the urine, these are seen as tubular erythrocyte casts. Since segmental GN affects only some glomeruli, 10 to 12 are required in the biopsy to distinguish focal from diffuse disease. In addition, only a segment of the glomerular tuft may be affected.

Crescentic GN. Glomeruli may also contain semicircular crescentic cells around the glomerular tuft, correlating with

treatment in patients with ESRD and suggest that it be used only in those with active disease.

\section{Renal transplant}

At the time of transplantation, patients receive a massive immunosupressive induction regimen consisting of antiCD25 antibody and triple conventional immunosuppressive drugs, usually a calcineurin inhibitor, antimetabolite, and prednisolone. Survival in transplant patients with vasculitis is not significantly different from that of other kidney transplant patients. ${ }^{15}$

Patients should not receive transplants until at least 12 months after induction of remission; patients who

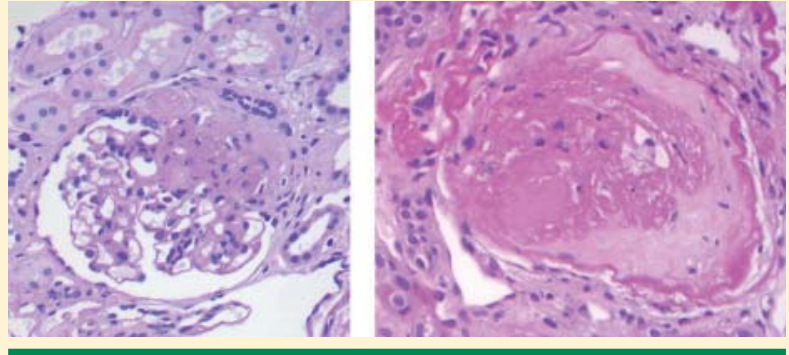

FIGURE 4. Fibrous crescent (left) and global sclerosis (right).

rapidly progressive GN. In a setting of active urinary sediment with erythrocyte casts, mouse-ear-shaped cells, some protein, and rapidly decreasing renal function, the inflammatory process within the glomerular capillary leads to rupture of the glomerular basement membrane and inflammatory cells in the vessel pass into the Bowman's capsule, forming a cellular crescent (Figure 3).

Fibrous crescents progressing to global sclerosis. As they age, cellular crescents become fibrous. Although fibrous crescents in the biopsy indicate a step toward scarring, and other extrarenal problems might indicate the need for immunosuppression, at this point the kidney will no longer benefit from immunosuppression. Kidney biopsy, therefore, provides important prognostic clues regarding whether immunosuppression will be helpful. With progression to global sclerosis, there is barely any capillary tuft evident and normal open capillaries. In this situation as well, immunosuppression is not indicated and will provide no benefit (Figure 4).

Immune histology. Focal segmental GN and crescents on biopsy alone are not diagnostic. Immunofluorescence is required to detect whether there are few or no deposits of complement and immunoglobulins, denoting pauci-immune AASV. In Goodpasture disease, there is a linear deposition of complement and immunoglobulins; in systemic lupus erythematosus and cryoglobulinemia, a more granular deposition appears. Immune histology is required to make such distinctions.

underwent transplant less than 12 months after remission had a mortality HR of $2.3(P<.05) .{ }^{16}$ Vasculopathy occurs more frequently in ANCA-positive patients, which leads to graft loss.

Graft loss due to recurrent vasculitis is also possible. Nachman et $\mathrm{al}^{17}$ found double the rate of infection for transplant compared with nontransplant AASV patients, but the rate of relapse was lower than the rate before transplant or for patients on dialysis. There was no significant difference in rates of relapse between patients with or without circulating ANCA at the time of transplant or between those having GPA, MPA, or renal-limited disease. ${ }^{18}$ 


\section{Cardiovascular risk}

Renal involvement in vasculitis increases cardiovascular morbidity. Vasculitis patients with renal involvement $(n=113)$ were matched with patients with chronic kidney disease and other contributing cardiovascular risk factors. ${ }^{18}$ After approximately 4 years of follow-up, the vasculitis patients had an HR of $2.23(P=.017)$ for cardiovascular events. AASV patients with the highest excess risk had histories of cardiovascular events (HR, 4), dialysis dependency (HR, 4.3), poor renal function at admission (HR, 0.977), and history of smoking (HR, 3.9).

\section{CONCLUSION}

Level of renal function at diagnosis is an important predictor of survival. Poor renal function correlates with mortality, especially in the elderly. Pathologic classification of renal vasculitis based on histopathology obtained from the kidney biopsy correlates closely with prognosis. About $60 \%$ of initially dialysis-dependent patients with active GN can regain independent renal function. Those on dialysis have a lower rate of relapse of active vasculitis than do those with independent renal function; patients with kidney transplants have the lowest rate of relapse. There is a doubling of infection rate in patients who have ESRD and who receive any form of renal replacement therapy. Lastly, renal involvement in AASV is an independent and serious contributor to risk for cardiovascular disease.

\section{REFERENCES}

1. Jennette JC, Falk RJ, Andrassy K, et al. Nomenclature of systemic vasculitides: proposal of an International Consensus Conference. Arthritis Rheum 1994; 37:187-192.

2. Booth AD, Almond MK, Burns A, et al; for the Pan-Thames Renal Research Group. Outcome of ANCA-associated renal vasculitis: a 5-year retrospective study. Am J Kidney Dis 2003; 41:776-784.

3. Reinhold-Keller E, Beuge N, Latza U, et al. An interdisciplinary approach to the care of patients with Wegener's granulomatosis: longterm outcome in 155 patients. Arthritis Rheum 2000; 43:1021-1032.

4. Weidner S, Geuss S, Hafezi-Rachti S, Wonka A, Rupprecht HD. ANCA-associated vasculitis with renal involvement: an outcome analysis [published online ahead of print April 6, 2004]. Nephrol Dial Transplant 2004; 19:1403-1411. doi: 10.1093/ndt/gfh161

5. Flossmann O, Berden A, de Groot K, et al; for the European Vasculitis Study Group. Long-term patient survival in ANCA-associated vasculitis [published online ahead of print November 24, 2010]. Ann
Rheum Dis 2011; 70:488-494. doi: 10.1136/ard.2010.137778

6. Hamour SM, Salama AD. ANCA comes of age-but with caveats. Kidney Int 2011; 79:699-701.

7. Harper L, Savage CO. ANCA-associated renal vasculitis at the end of the twentieth century — a disease of older patients [published online ahead of print December 21, 2004]. Rheumatology 2005; 44:495-501. doi: 10.1093/rheumatology/keh522

8. Berden AE, Ferrario F, Hagen EC, et al. Histopathologic classification of ANCA-associated glomerulonephritis [published online ahead of print July 8, 2010]. J Am Soc Nephrol 2010; 21:1628-1636. doi: 10.1681/ASN.2010050477

9. de Groot K, Harper L, Jayne DRW, et al; for the EUVAS (European Vasculitis Study Group). Pulse versus daily oral cyclophosphamide for induction of remission in antineutrophil cytoplasmic antibody-associated vasculitis: a randomized trial. Ann Intern Med 2009; 150:670-680.

10. Harper L, Morgan MD, Walsh M, et al; on behalf of the EUVAS Investigators. Pulse versus daily oral cyclophosphamide for induction of remission in ANCA-associated vasculitis: long-term follow-up [published online ahead of print November 29, 2011]. Ann Rheum Dis 2012; 71:955-960. doi: 10.1136/annrheumdis-2011-200477

11. Little MA, Nightingale P, Verburgh CA, et al; for the European Vasculitis Study (EUVAS) Group. Early mortality in systemic vasculitis: relative contribution of adverse events and active vasculitis [published online ahead of print July 1, 2009]. Ann Rheum Dis 2010; 69:1036-1043. doi: 10.1136/ard.2009.109389

12. Jayne DR, Gaskin G, Rasmussen N, et al; for the European Vasculitis Study Group. Randomized trial of plasma exchange or highdosage methylprednisolone as adjunctive therapy for severe renal vascultis [published online ahead of print June 20, 2007]. J Am Soc Nephrol 2007; 18:2180-2188. doi: 10.1681/ASN.2007010090

13. Lionaki S, Hogan SL, Jennette CE, et al. The clinical course of ANCA small-vessel vasculitis on chronic dialysis [published online ahead of print June 17, 2009]. Kidney Int 2009; 76:644-651. doi: 10.1038/ki.2009.218

14. Weidanz F, Day CJ, Hewins P, Savage CO, Harper L. Recurrences and infections during continuous immunosuppressive therapy after beginning dialysis in ANCA-associated vasculitis. Am J Kidney Dis 2007; 50:36-46.

15. Schmitt WH, van der Woude FJ. Organ transplantation in the vasculitides. Curr Opin Rheumatol 2003; 15:22-28.

16. Little MA, Hassan B, Jacques S, et al. Renal transplantation in systemic vasculitis: when is it safe [published online ahead of print July 13, 2009]? Nephrol Dial Transplant 2009; 24:3219-3225. doi: $10.1093 /$ ndt/gfp347

17. Nachman PH, Segelmark M, Westman K, et al. Recurrent ANCAassociated small vessel vasculitis after transplantation: a pooled analysis. Kidney Int 1999; 56:1544-1550.

18. Morgan MD, Turnbull J, Selamet U, et al. Increased incidence of cardiovascular events in patients with antineutrophil cytoplasmic antibody-associated vasculitides: a matched-pair cohort study. Arthritis Rheum 2009; 60:3493-3500.

Correspondence: Kirsten de Groot, MD, Medizinische Klinik III, Klinikum Offenbach, KfH Nierenzentrum Offenbach, Starkenburgring 66, 63069 Offenbach, Germany; kirsten@de-groot.de 


\title{
Pulmonary disease in small-vessel vasculitis
}

\author{
THOMAS R. GILDEA, MD, MS
}

Head, Section of Bronchoscopy, Transplant Center, Respiratory Institute, Cleveland Clinic, Cleveland, OH

\section{ABSTRACT}

Diagnosis of the pulmonary manifestations of small-vessel vasculitis requires attention to detail, judicious use of imaging technology, and awareness of disorders that can mimic or masquerade as pulmonary vasculitis. Treatment should begin with pharmacologic intervention to manage the underlying inflammatory disorder. Dilation procedures and, in rare cases, surgery may be needed to resolve airway stenosis.

T he pulmonary manifestations of small-vessel vasculitis are nonspecific and often overlap with other conditions. Consequently, the diagnosis and management of pulmonary vasculitis are complex and require special attention to detail. This article reviews clinical experience with vasculitis as it manifests in the pulmonary setting, with the goal of providing a sound clinical approach to diagnosis and management.

\section{DIAGNOSTIC CONSIDERATIONS}

Accurate diagnosis is enhanced with imaging technology, judicious use of bronchoscopy, and awareness of disorders that mimic or masquerade as pulmonary vasculitis. The diagnosis can be approached on the basis of pattern recognition. For example, microscopic polyangiitis (MPA) is characterized solely by alveolar hemorrhage syndrome. However, other diagnostic possibilities must be considered, such as infection, acute respiratory distress syndrome, and complications of medicines. The hallmark manifestation of granulomatosis with polyangiitis (GPA [Wegener's granulomatosis]) is necrotizing granulomatous inflammations, but the pulmonary manifestations can include nodules, cavitary masses, airway stenosis, and alveolar hemorrhage. Asthma with eosinophilia is the distinguishing feature of eosinophilic GPA (Churg-Strauss syndrome, EGPA), and Goodpasture syndrome involves deposition of complement and immunoglobulins.

\section{The use of imaging}

The best imaging tool for suspected pulmonary vasculitides is high-resolution computed tomography (CT). As a general rule, CT for patients with suspected vasculitis should be ordered without contrast medium as contrast is not needed to assess the lung parenchyma. Vasculitis patients often have renal insufficiency, and contrast-free CT will help protect the kidneys. Another option, which will enhance evaluation of the distribution and location of pulmonary disease, is multiplanar reconstructions of images with virtual bronchoscopy or airway reconstruction. Certain findings on imaging will help to differentiate the vasculitides from one another as well as from mimicking diagnoses.

Eosinophilic GPA. Chest images of patients with EGPA appear as patchy, nonsegmental, often peripheral consolidations of ground-glass opacity. These tend to reside in all lobes of the lungs, close to the surface and occasionally accompanied by septal markings.

Microscopic polyangiitis. Although classically a disease of alveolar hemorrhage, MPA often does not manifest with hemoptysis. Approximately one-third of patients with MPA do not cough up blood, even after a large amount of hemorrhage directly into the parenchyma. Patients may present with nonspecific symptoms such as fatigue and shortness of breath. Chest imaging will enhance diagnostic accuracy, particularly when considered in conjunction with laboratory test results. MPA patients usually have low hematocrit levels and may actually have an increased diffusing capacity of the lung for carbon monoxide (DLCO).

Granulomatosis with polyangiitis. This form of vasculitis has characteristic nodules, cavitary lesions, and, in the worst cases, multifocal masses in the lungs. These can be identified with contrast-free CT, with examination for possible airway involvement.

Multiple lung cavity nodules and pronounced airway narrowing are significant diagnostic clues for GPA. Nodules up to $10 \mathrm{~cm}$ in diameter tend to be near subpleural and peripheral areas. Microbes and fungus may complicate the nodules' primary presentation. While bronchoscopy may be helpful with imaging, surgical biopsy remains the gold standard to rule out infections.

The disease may be multifocal, occurring outside the lungs from the larynx to bronchi and anywhere in the lung. Subglottic stenosis caused by inflammation and scarring affects $16 \%$ of patients with GPA, but it also often develops independently of other features of GPA and may have its own course independent of systemic symptoms. ${ }^{1}$
Dr. Gildea reported teaching and speaking services for SuperDimension Inc.

This article was developed from an audio transcript of Dr. Gildea's presentation at the "New Directions in Small-Vessel Vasculitis: ANCA, Target Organs, Treatment, and Beyond" symposium held at Cleveland Clinic on May 4, 2011. The transcript was formatted and edited by Cleveland Clinic Journal of Medicine staff for clarity and conciseness, and was then reviewed, revised, and approved by Dr. Gildea.

doi:10.3949/ccjm.79.s3.06 


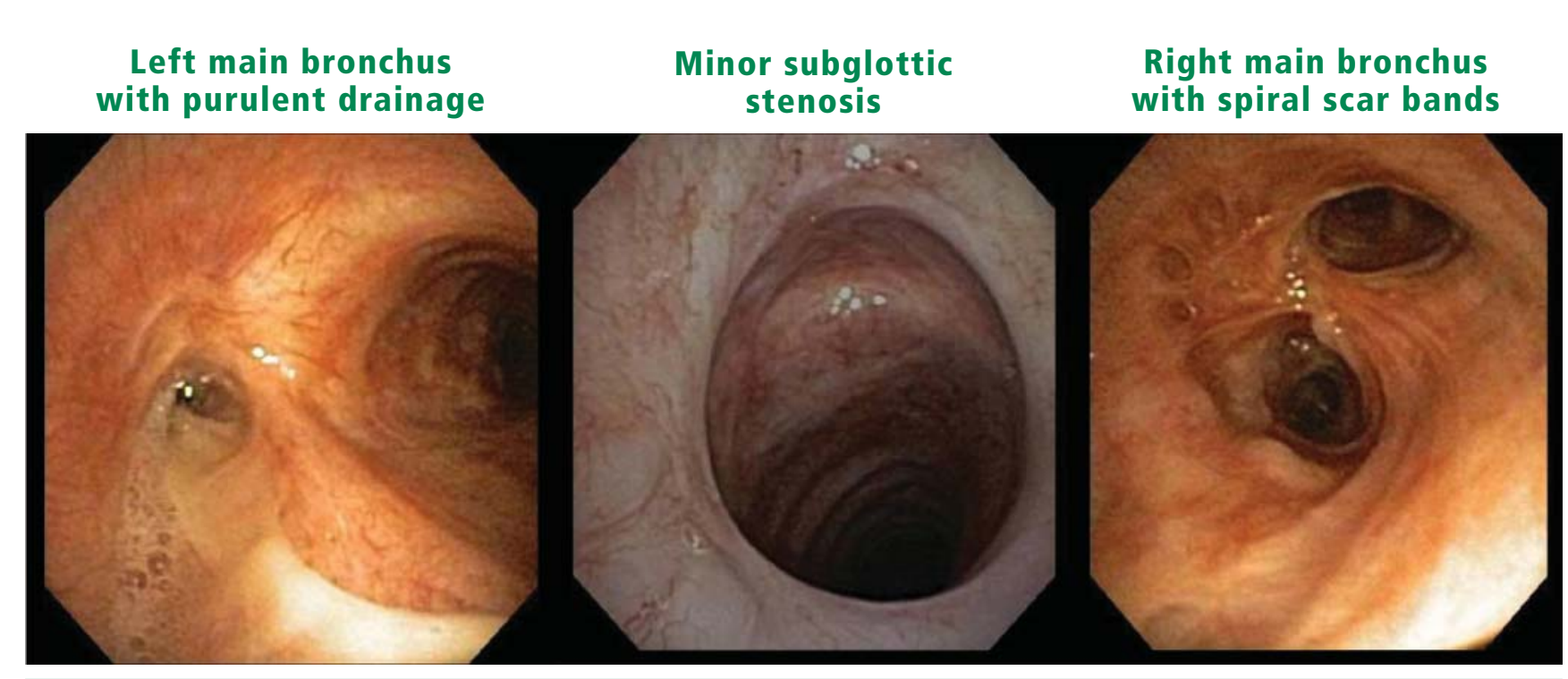

FIGURE. Multiple levels of endobronchial disease are evident in a patient who has minor subglottic stenosis as well as a severely occluded left main bronchus with airway wall thickening and scar bands in the right main bronchus. The left main bronchus requires endobronchial therapy to relieve postobstructive pneumonia.

\section{Bronchoscopy}

Bronchoscopy is a relatively low-risk way to assess airways and nodules, but it has had a limited role in the diagnosis of nonfocal interstitial lung disease and rheumatologic lung disease in general. New technologies that augment traditional bronchoscopy and enhance its utility for diagnosis for focal entities are described below.

Electromagnetic navigation bronchoscopy (ENB) uses electromagnetic technology to localize and guide a catheter through the bronchial pathways. With the help of a virtual, 3-dimensional bronchial map reconstructed from a chest CT, the clinician can navigate to a desired location within the lung for biopsy and diagnosis of pulmonary nodules. The result is a diagnostic yield per nodule of nearly $80 \%{ }^{2}$ Seijo et al showed that diagnostic yields by ENB increase with the presence of the bronchus sign, or a bronchus leading directly to a peripheral lung lesion, as viewed on CT imaging. ${ }^{2}$ If nodules are bronchocentric, or surround airways, there is greater likelihood of reaching a diagnosis without resorting to surgery.

In peripheral radial ultrasound, a catheter is threaded through another catheter sheath in order to visualize the lesion. This technology can precisely localize lung lesions and often give some clues about the final pathology.

Bronchoscopic confocal fluorescence microscopy ${ }^{3}$ is a new form of microscopy that uses a fiberoptic miniprobe instead of an objective lens. High-quality images are achieved by the use of autofluorescence. Researchers have used the technology to detect changes in the respiratory bronchioles and other structures, but a clear atlas of many disease states does not yet exist. Oddly, endobronchial GPA images have been catalogued. ${ }^{3}$
Virtual bronchoscopy is a 3-dimensional image reconstruction and display technique that converts standard CT images into multiplanar images, which can be stacked. Virtual bronchoscopy augments conventional CT because of its ability to enhance detection in the subglottic region and more accurately measure stenosis. ${ }^{4}$ The technique cannot replace traditional bronchoscopy, however, because mucus and secretions can appear as abnormalities and cause false-positive results.

Airway examination can often reveal multiple levels of airway disease in a single patient (Figure).

\section{Detecting mimics}

Diagnoses that masquerade as EGPA include chronic eosinophilic pneumonia, bronchiolitis obliterans with organizing pneumonia, and other interstitial lung diseases. Allergic bronchopulmonary aspergillosis-an asthma syndrome sometimes associated with eosinophilia and high immunoglobulin-E levels_also mimics EGPA. This diagnostic possibility is particularly relevant if the patient is taking immunosuppressive agents or corticosteroids.

Although alveolar hemorrhage is the sole pulmonary manifestation of MPA, the diagnosis is not limited to MPA alone. Alveolar hemorrhage may have other causes, including infection or acute respiratory distress syndrome. Bronchial lavage is recommended for accurate diagnosis, with the introduction of successive volumes of saline into the lungs and examination for increasing amounts of heme in each of the aliquots of alveolar lavage fluid.

Several diagnoses can mimic GPA. Many infections, including those caused by mycobacteria and Cryptococcus, can mimic endobronchial GPA. Biopsy of all new 
ulcers is recommended to minimize the possibility of missing these diagnoses. Tuberculosis in its latent form can closely resemble scarred GPA. Other mimickers of cavitary lung lesions can include metastatic melanoma, metastatic renal and thyroid cancers, squamous cell carcinoma, and rheumatoid arthritis with necrobiotic nodules that open in the lungs.

\section{TREATMENT STRATEGIES}

\section{Medications}

Although many patients with GPA are surgical candidates because of dyspnea related to fixed endobronchial or endotracheal obstructions, any surgical treatment carries the risk of inciting further flares. Treatment should focus first on mitigating the systemic inflammatory disorder with pharmacologic intervention. Standard pharmacologic therapy includes corticosteroids, azathioprine, cyclophosphamide, and rituximab. Patients with subglottic stenosis are frequently unresponsive to standard immunosuppressive therapy (glucocorticoids in combination with a cytotoxic agent). ${ }^{1}$

\section{Surgical reconstruction}

When medication falls short and surgery is needed to reverse strictures, a number of tools are at our disposal. Some involve heat, such as laser, cauterization, and argon plasma coagulation. In argon plasma coagulation, a jet of ionized argon gas (plasma) is directed through a probe passed through an endoscope. Other techniques rely on cold: cryoprobes, microdebriders, and rigid scissors. In general, freeze therapies cause less scarring than heat therapy. With any surgical technique, there is risk of scars that will contract and cause structural collapse, resulting in restenosis.

\section{Dilation}

The high rate of stenosis relapse has spurred interest in alternatives to surgical treatment. One of these, dilation via endoscopy, also may mitigate the wound healing process. Other techniques for clearing the obstructed area include rigid bronchoscopy, the use of bougies (increasingly larger dilators), and balloon dilation. Balloon dilation has some advantages over the other techniques. It permits maximal radial direction and pressure, causes less damage to trachea wall mucosa, and achieves better overall results; however, the procedure usually needs to be repeated. ${ }^{5}$ It must be done quickly, and it requires flawless communication between the otolaryngologist or pulmonologist and anesthesiologist in order to stabilize the airway below the vocal cords.

\section{Intratracheal dilation-injection therapy}

Dilation can be augmented with glucocorticoid injections. In 1991, researchers at the National Institutes of Health utilized a combination dilation-injection therapy for 20 patients who had GPA and subglottic stenosis. ${ }^{1}$ Patients were first treated with mercury-filled dilators coated with $1 \%$ triamcinolone cream. Methylprednisolone acetate was then injected into the stenotic area. None of the patients treated with intratracheal dilation-injection therapy required a tracheostomy and six who already had tracheostomies were decannulated. In contrast, 56\% of patients who received standard immunosuppressive therapy and no intratracheal dilation-injection therapy required tracheostomy. Intratracheal dilation-injection therapy is considered a safe and effective treatment of GPA-associated subglottic stenosis and, in the absence of major organ disease activity, could be used without systemic immunosuppressive agents.

Mitomycin- $\mathrm{C}$ is a controversial alternative to corticosteroids during dilation. Mitomycin- $\mathrm{C}$ is an alkylating agent that inhibits fibroblast proliferation and extracellular matrix protein synthesis, with the potential for reduced scarring. In a recent trial of 26 patients, two doses given 3 to 4 weeks apart reduced the rate of stenosis for 2 to 3 years compared with a single dose. ${ }^{6}$ Restenosis occurred in both groups, however, and after 5 years, the relapse rates were the same.

\section{Nd:YAG laser photoresection versus endobronchial electrosurgery}

One of the most effective therapies for treating obstructive lesions is Nd:YAG laser photoresection (LPR) in which a laser that utilizes the crystal neodymium-doped yttrium aluminum garnet $\left(\mathrm{Nd}: \mathrm{Y}_{3} \mathrm{Al}_{5} \mathrm{O}_{12}\right)$ is paired with a flexible bronchoscope. The procedure can produce favorable outcomes, ${ }^{7}$ but it has not gained favor because of perceptions that the lasers require rigid bronchoscopy, expensive equipment, and special training. There are also concerns about complications.

The lower-cost endobronchial electrosurgery (EBES) also failed to gain acceptance because of cumbersome delivery systems and complications associated with power units. Recently, engineers have spawned a new generation of electrosurgical devices, prompting renewed interest in EBES.

A recent study compared LPR and EBES in patients who represented 118 evaluations for LPR. ${ }^{8}$ Forty percent were considered amenable to EBES and so did not go on to receive the more costly LPR. Of those, $89 \%$ achieved success in alleviating the obstruction. The authors concluded that EBES can potentially eliminate the need for LPR in 36\% of procedures, and that it could achieve significant savings in cost and time. We use these ablative therapies only in dire circumstances; we use non-heat-based therapies, including repeated dilation, prior to considering use of other therapies.

\section{Cryotherapy}

Cryotherapy spray was initially thought to have great therapeutic potential, but the high pressures of the spray caused complications. This modality remains under investigation, however. Some probe-based cryotherapy 
techniques have been effective anecdotally. These use a metal-tipped probe attached to a cryogen; the JouleThompson effect causes delayed tissue destruction.

\section{Stents}

A small number of case reports note patient improvement after stenting., ${ }^{9,10}$ We use stents in rare circumstances, but because complications are frequent and sometimes severe, we consider stenting a last-resort option. In 2005, the US Food and Drug Administration mandated a Black Box warning against the use of metallic stents in patients who have benign tracheal strictures.

\section{Multimodality therapies}

In general, when intervention is required to salvage airways, a combination of dilation and steroid injection with or without topical mitomycin-C is standard. We try to avoid use of thermal therapy with laser or electrocautery because of the risk of exuberant inflammation and restenosis from thermal injury. No specific standard of care exists in these cases; reliance on clinical judgment is critical because of the presentation and variety of airway lesions. Further, no large-scale randomized trials exist to guide therapy, so it is best to work with a multidisciplinary team whose members have experience in managing these complex patients.

\section{CONCLUSION}

The differential diagnosis of pulmonary manifestations of small-vessel vasculitis is complex. Several diagnoses can mimic various forms of pulmonary vasculitis, and the manifestations and symptoms often overlap with other organ systems.

Imaging is useful for analysis of common patterns of small and midsize vasculitis, although the results may be confounded by disorders that mimic pulmonary vasculitis. To enhance diagnostic accuracy, laboratory and clinical findings should be considered along with images. Ideally, treatment will be minimally destructive and mucosa-sparing. Dilation therapies can be augmented with corticosteroid injections or, possibly, mitomycin-C.

\section{REFERENCES}

1. Langford CA, Sneller MC, Hallahan CW, et al. Clinical features and therapeutic management of subglottic stenosis in patients with Wegener's granulomatosis. Arthritis Rheum 1996; 39:1754-1760.

2. Seijo LM, de Torres JP, Lozano MD, et al. Diagnostic yield of electromagnetic navigation bronchoscopy is highly dependent on the presence of a bronchus sign on CT imaging: results from a prospective study. Chest 2010; 138:1316-1321.

3. Filner JJ, Bonura EJ, Lau ST, et al. Bronchoscopic fibered confocal fluorescence microscopy image characteristics and pathologic correlations. J Bronchol Intervent Pulmonol 2011; 18:23-30.

4. Summers RM, Aggarwal NR, Sneller MC, et al. CT virtual bronchoscopy of the central airway in patients with Wegener's granulomatosis. Chest 2002; 121:242-250.

5. Schokkenbroek AA, Franssen CFM, Dikkers FG. Dilatation tracheoscopy for laryngeal and tracheal stenosis in patients with Wegener's granulomatosis. Eur Arch Otorhinolaryngol 2008; 265:549-555.

6. Smith ME, Elstad M. Mitomycin C and the endoscopic treatment of laryngotracheal stenosis: are two applications better than one? Laryngoscope 2009; 119: 272-283.

7. Shvero J, Shitrit D, Koren R, Shalomi D, Kramer MR. Endoscopic laser surgery for subglottic stenosis in Wegener's granulomatosis. Yonsei Med J 2007; 48:748-753.

8. Coulter TD, Mehta AC. The heat is on: impact of endobronchial electrosurgery on the need for Nd-YAG laser photoresection. Chest 2000; 118:516-521.

9. Tierman J, Shah C, Elborn JS. Successful stenting in endobronchial Wegener's granulomatosis. Ulster Med J 2006; 75:155-157.

10. Daum TE, Specks U, Colby TV, et al. Tracheobronchial involvement in Wegener's granulomatosis. Am J Respir Crit Care Med $1995 ; 151: 522-526$.

Correspondence: Thomas R. Gildea, MD, MS, Transplant Center, Respiratory Institute, Cleveland Clinic, 9500 Euclid Avenue, A90, Cleveland, OH 44195; gildeat@ccf.org 


\title{
Ocular manifestations of small-vessel vasculitis
}

\author{
JAMES A. GARRITY, MD \\ Whitney and Betty MacMillan Professor, Department of Ophthalmology, Mayo Clinic, Rochester, MN
}

\section{ABSTRACT}

Ophthalmic manifestations of vasculitis can be orbital, ocular (affecting the globe), or intraocular. Orbital inflammation manifests as sudden onset of pain, erythema, and proptosis, and can be sight-threatening. In the globe, red eye is typical in both episcleritis and scleritis. Episcleritis is usually otherwise asymptomatic with blanching upon instillation of topical phenylephrine, whereas scleritis is painful and does not blanch. Infectious and rheumatic diseases are present in nearly $50 \%$ of patients with scleritis. The symptoms of keratitis are similar to those of scleritis; superficial keratitis is benign but peripheral ulcerative keratitis can be sight-threatening. Anterior uveitis is the most frequent ocular manifestation of Behçet disease. Approximately $30 \%$ of patients with granulomatosis with polyangiitis (Wegener's granulomatosis) have ocular involvement, with orbital disease being most common. With ophthalmic manifestations of vasculitis, tissue biopsy of any site that is amenable to biopsy is recommended. Biopsy must be interpreted within the context of treatment.

W e have long understood that vasculitic conditions have various clinical manifestations. The Chapel Hill Consensus Conference classification of systemic vasculitis in $1994^{1}$ contributed significantly to our understanding of the spectrum of vasculitides and their manifestations, enhancing our diagnostic ability and the likelihood of appropriate treatment.

The ophthalmic manifestations of vasculitis are protean and nonspecific, and should be considered in the overall context of the disease. Patients should be evaluated with the following questions in mind:

- Are the manifestations related to the vasculitis itself?

- Are the manifestations a result or complication of therapy?

- Are the manifestations signs of a completely unrelated and superimposed condition?

This article reviews the three areas of ocular inflammation related to vasculitis and comments on the role of tissue biopsy in the management of these patients.

\section{THREE AREAS OF OCULAR INFLAMMATION}

\section{Orbital inflammation}

Orbital disease can affect the lacrimal gland (inflammatory dacryoadenitis), extraocular muscles (orbital myositis), and the orbital soft tissues (inflammatory orbital pseudotumor). Orbital inflammation is characterized by relatively sudden onset (within days) of pain, erythema, and proptosis. Diplopia and visual loss from either compression or inflammation of the optic nerve or nerve sheath may be present. Depending upon the structures involved and the degree of involvement, orbital inflammation can be sight-threatening.

Either computed tomography or magnetic resonance imaging should be performed to assess orbital or extraorbital involvement. The orbital structures are particularly amenable to biopsy, which, in this author's opinion, should be performed whenever possible. The biopsy may need to be interpreted within the context of previous or concurrent immunosuppressive therapy, which can alter the histologic picture, minimize inflammation, and make detection of vasculitis difficult. In addition to identifying inflammation, biopsy helps to identify fungal infection or lymphoma that can follow prolonged immunosuppressive therapy.

Treatment of orbital inflammation requires corticosteroid therapy or some other type of systemic immunosuppression.

\section{Ocular, or globe, inflammation}

The globe has three areas subject to inflammation: episcleral tissues (episcleritis), sclera (scleritis), and the cornea (keratitis) (Figure 1).

Episcleritis: observation or topical therapy. Episcleritis usually manifests as an otherwise asymptomatic red eye with typical sector-shaped inflammation. Pain is generally not an issue, although patients often report that the eye does not feel normal. Vision is unaffected and there is no potential threat to sight.

The slit-lamp examination shows dilated vessels in the episcleral tissues that blanch after instillation of a drop of $10 \%$ phenylephrine. Simple observation may be the best management course, but topical nonsteroidal antiinflammatory drugs (NSAIDs) or topical corticosteroids may help some patients who have discomfort.
Dr. Garrity reported that he has no financial interests or relationships that pose a potential conflict of interest with this article.

This article was developed from an audio transcript of Dr. Garrity's presentation at the "New Directions in Small-Vessel Vasculitis: ANCA, Target Organs, Treatment, and Beyond" symposium held at Cleveland Clinic on May 4, 2011.
The transcript was formatted and edited by Cleveland Clinic Journal of Medicine staff for clarity and conciseness, and was then reviewed, revised, and approved by Dr. Garrity.

doi:10.3949/ccjm.79.s3.07 


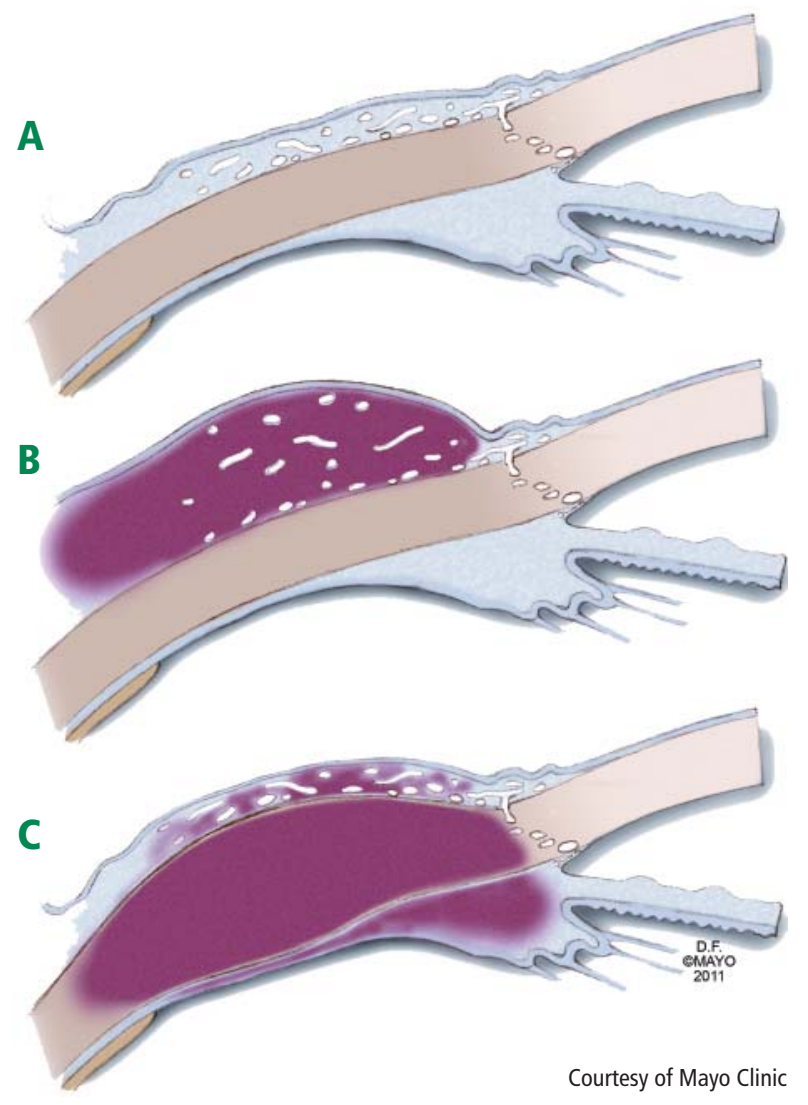

FIGURE 1. Cross-sectional anatomy of the eye. (A) Normal episcleral and scleral tissues. (B) Episcleritis with inflammation and thickening of the episcleral tissues. (C) Scleritis with inflammation and swelling of the sclera and secondary peripheral corneal swelling, episcleral swelling, and ciliary body swelling.

There is probably a spectrum of disease in that some patients may have either severe episcleritis or mild scleritis (Figure 1B). At times it can be difficult to differentiate between severe episcleritis and mild scleritis. Although scleritis generally requires systemic therapy, topical therapy is justified for mild scleritis. Episcleritis is associated with systemic disease in approximately $36 \%$ of patients. ${ }^{2-4}$

Scleritis: may be sight-threatening; requires systemic therapy. Scleritis characteristically presents with intense pain and a red eye., ${ }^{3,5-7}$ Patients may be sensitive to light and their vision may be compromised. Cataracts and glaucoma can complicate the course of scleritis.

With slit-lamp examination, the redness does not blanch upon instillation of topical 10\% phenylephrine as it does with episcleritis. The adjacent cornea may also be affected (Figure 1C). Healed scleritis leaves an area of thinned sclera that appears as a visible blue spot, so if the patient's history includes red eye with pain and a blue area is visible, the clinician can be confident that a prior episode of scleritis occurred.

Scleritis can be anterior or posterior, and the implica- tions are slightly different for each type. Anterior scleritis can be subclassified as diffuse, nodular, or necrotizing. The necrotizing type can be characterized by painful inflammation or, in the case of scleromalacia perforans, no inflammation and no pain. Posterior scleritis may have minimal pain.

Akpek et $\mathrm{al}^{5}$ reported on a group of 243 patients with scleritis (average age, 52 years; range, 5 to 93 years) who were followed for an average of 1.7 years (range, 0 to 16.6 years). An associated medical condition was present in 107 (44\%) patients. Rheumatologic conditions accounted for $37 \%$, with rheumatoid arthritis being most common; infectious disease, with herpes zoster ophthalmicus being most common, accounted for $7 \%$. Of those with an associated medical condition, $78 \%$ had been diagnosed previously; the remaining $22 \%$ were diagnosed at presentation or the condition developed during follow-up.

Treatment typically requires systemic therapy with NSAIDs, but more often oral or intravenous corticosteroids or even methotrexate, mycophenolate mofetil, cyclophosphamide, or rituximab may be required. Patients with antineutrophil cytoplasmic antibody (ANCA)-positive disease may require more intensive therapy than those with ANCA-negative disease.

Keratitis: may be sight-threatening. Patients with keratitis should be evaluated in the same spirit as patients with scleritis (Figure 1C). Although many patients may have superficial keratitis, which is often related to a dry eye and has no prognostic significance, deep or peripheral ulcerative keratitis is not only consistent with systemic vasculitis but also sight-threatening. Symptoms similar to those observed with scleritis typically include severe pain and photophobia and, as with scleritis, treatment usually involves systemic therapy.

\section{Intraocular inflammation}

Vascular involvement. Vasculitic involvement of blood vessels within and around the eye can produce several different clinical pictures. Within the eye are two separate intraocular circulations, the retinal vessels and the choroid circulation. Retinal vessel involvement can affect the retinal arteries with thrombotic occlusions, the veins with phlebitis, or both. Retinal artery occlusions take the form of a branch or central retinal artery occlusion that infarcts corresponding portions of the retina, leading to loss of vision. The optic disc is also subject to vascular interruption of the posterior ciliary arteries, which constitute a separate branch off the ophthalmic artery. This condition produces an ischemic optic neuropathy and results in loss of vision (Figure 2).

There is no specific treatment for the eye other than treating the underlying condition. Vascular occlusions can sometimes give rise to neovascularization and patients should be followed for this possibility. As with a central nervous system ischemic event, recovery can be variable.

Uveitis. The term "uvea," derived from the Greek 
word for grape, describes the shape of the iris, ciliary body, and choroid. Uveitis is a generic term for intraocular inflammation affecting any or all of these structures.

Iritis, or anterior uveitis, is a frequent accompaniment of keratitis or scleritis. Primarily uveitic involvement with retinal vessel vasculitis involving both arteries and veins is uncommon in general but typical of Behçet disease, especially if a hypopyon uveitis is present.

Anterior uveitis can be treated with topical corticosteroids and cycloplegic drugs, but middle and posterior uveitis almost always requires systemic therapy. Most recently, use of anti-tumor necrosis

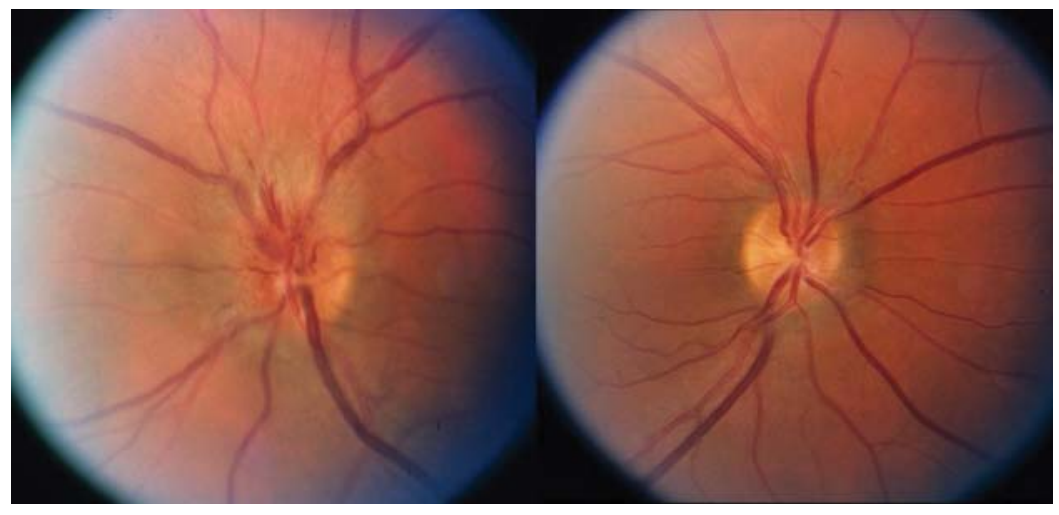

FIGURE 2. Ischemic optic neuropathy. Appearance of ischemic optic neuropathy (left panel) versus normal fellow eye (right panel). factor- $\alpha$ drugs has been effective in treating Behçet uveitis. ${ }^{8}$ The visual prognosis with Behçet disease remains guarded.

\section{GRANULOMATOSIS WITH POLYANGIITIS: EYE INVOLVEMENT IS COMMON}

In terms of specific small-vessel vasculitic diseases that affect the eye, granulomatosis with polyangiitis (GPA [Wegener's granulomatosis]) is the quintessential condition. In data obtained from the Wegener Granulomatosis Support Group, ${ }^{9}$ eye involvement was noted at presentation in 211 of 701 patients (30\%), and during the course of their disease an additional 147 patients developed eye involvement. From the time of initial presentation through the course of follow-up, 359 of the 701 patients (51\%) eventually had some type of eye involvement.

In a series of patients seen at the Mayo Clinic, ${ }^{10}$ orbital inflammatory disease and scleritis were the two most frequent manifestations of eye involvement with GPA. Orbital involvement typically presents with pain, erythema, swelling, and proptosis. Varying degrees of ptosis, diplopia, or visual loss may also be present. Imaging may show an infiltrate that is usually adjacent to the maxillary or ethmoid sinus. This same process can affect the superior temporal orbital quadrant, an area apart from any sinus, and involve the lacrimal gland.

\section{BIOPSY IS ADVISED}

Biopsy, either incisional, at times to include debulking, or excisional if possible, is recommended to establish a diagnosis or aid in the selection of therapy. Orbital disease has been observed to progress in patients who are receiving maintenance therapy with methotrexate and have no evidence of systemic disease activity. Acute and chronic inflammation with evidence of active vasculitis is usually seen histologically. Personal observations suggest that intraorbital corticosteroid injection followed by rituximab has been effective therapy for this limited subset of patients. Diagnostic biopsies often must be

interpreted in light of partial treatment, making histopathologic diagnosis challenging at times. Biopsy is important for exclusion of lymphoproliferative disease or fungal infection.

\section{CONCLUSION}

Underlying vasculitis might play a role in patients with nonspecific ocular presentations. It is essential that the ophthalmologist collaborate with a specialist in vasculitis (and vice versa) for evaluation and subsequent therapy, which often involves some form of immunosuppression.

\section{REFERENCES}

1. Jennette JC, Falk RJ, Andrassy K, et al. Nomenclature of systemic vasculitides: proposal of an International Consensus Conference. Arthritis Rheum 1994; 37:187-192.

2. Pavesio CE, Meier FM. Systemic disorders associated with episcleritis and scleritis. Curr Opin Ophthalmol 2001; 12:471-478.

3. Jabs DA, Mudun A, Dunn JP, Marsh MJ. Episcleritis and scleritis: clinical features and treatment results. Am J Ophthalmol 2000; 130:469-476.

4. Akpek EK, Uy HS, Christen W, Gurdal C, Foster CS. Severity of episcleritis and systemic disease association. Ophthalmology 1999; 106:729-731.

5. Akpek EK, Thorne JE, Qazi FA, Do DV, Jabs DA. Evaluation of patients with scleritis for systemic disease. Ophthalmology 2004; 111:501-506.

6. McCluskey PJ, Watson PG, Lightman S, Haybittle J, Restori M, Branley M. Posterior scleritis: clinical features, systemic associations, and outcome in a large series of patients. Ophthalmology 1999; 106:2380-2386.

7. Riono WP, Hidayat AA, Rao NA. Scleritis: a clinicopathologic study of 55 cases. Ophthalmology 1999; 106:1328-1333.

8. Tabbara KF, Al-Hemidan AI. Infliximab effects compared to conventional therapy in the management of retinal vasculitis in Behçet disease [published online ahead of print October 17, 2008]. Am J Ophthalmol 2008; 146:845-850. doi: 10.1016/j.ajo.2008.09.010

9. Abdou NI, Kullman GJ, Hoffman GS, et al. Wegener's granulomatosis-survey of 701 patients in North America: changes in outcome in the 1990s. J Rheumatol 2002; 29:309-316.

10. Bullen CL, Liesegang TJ, McDonald TJ, DeRemee RA. Ocular complications of Wegener's granulomatosis. Ophthalmology 1983; 90:279-290.

Correspondence: James A. Garrity, MD, Department of Ophthalmology, Mayo Clinic, 200 First Street SW, Rochester, MN 55905; Garrity.james@mayo.edu 